Friedhelm Neidhardt

\title{
Institution, Organisation, Interaktion
}

\author{
Funktionsbedingungen des Wissenschaftsrats
}

Mit einem Bund-Länder-Abkommen 1957 gegründet, überstand der deutsche Wissenschaftsrat den Wechsel der Regierungen und die schwankenden Konjunkturen der Wissenschaftspolitik, ohne seine Grundstrukturen wesentlich zu verändern. Ihm ist es auch gelungen, seine von Anfang an vorhandene Reputation zu festigen und auszubauen. Im Hinblick auf einzelne Entscheidungen, die er getroffen hat, ist der Wissenschaftsrat zwar keineswegs unumstritten, aber er gilt selber als eine fest etablierte Institution. Er ist Teil des »komplexen Musters multipler Interessenvermittlungen ", das sich in der Bundesrepublik in der Nachkriegsgeschichte über die einzelnen Politikfelder hinweg entwickelte. ${ }^{1}$ In der Wissenschaftspolitik stellt er »die wohl wichtigste koordinierende Instanz $\ll^{2}$ dar, ein $»$ integrative model $\ll^{3}$ und in dieser Rolle ein »international einzigartiges Beispiel «. ${ }^{4}$ Seine Wertschätzung geht so weit, dass die großen Parteien des Bundestags zurzeit daran denken, für den Bildungsbereich nach dem »Vorbild des Wissenschaftsrats « einen »Bildungsrat « neu einzurichten. ${ }^{5}$ Dass Ernst-Ludwig Winnacker, ein ehemaliger Präsident der Deutschen Forschungsgemeinschaft, den Wissenschaftsrat kürzlich für entbehrlich hielt, wurde auffällig vor allem angesichts der geschlossenen Front, mit der die wissenschaftspolitisch einflussreichsten Akteure seinen Vorstoß konterten. ${ }^{6}$

Offenkundig ist einer insgesamt relativ schwachen Organisation eine relativ starke Institutionalisierung gelungen. Als relativ schwach erscheint die Organisation des Wissenschaftsrats insofern, als seine Strukturen nur im Hinblick auf bestimmte konstitutive Rahmenbedingungen formell festgelegt sind. In diesem Rahmen wirkt er wie ein großer Versammlungs- und Verständigungsbetrieb mit zahlreichen informellen Usancen, an die sich zu gegebener Zeit die Kölner Geschäftsstelle, sein professioneller Kern, erinnert. ${ }^{7}$ Regelmäßig mobilisiert er weit über seine kaum defi-

1 Lepsius 1990, S. $71 \mathrm{ff}$.

2 Braun 1997, S. 309.

3 Stucke 2011, S. 150

4 Weingart, Lentsch 2008, S. 36.

5 Vgl. Thies 2011, S. 3; ähnlich Burchardt 2011.

6 Vgl. zu einem Interview mit Winnacker die Presseinformation der duz-Redaktion vom 21. März 2012. Über die »heftigen Gegenreaktionen « berichtet der Tagesspiegel am 22. März 2012. Zu den Vorstellungen Winnackers inhaltlich weiter unten Fußnote 20.

7 Beispiele berichtet ein ehemaliger Generalsekretär der Geschäftsstelle des Wissenschaftsrats (Benz 1996, S. 1674 f.)

Leviathan, 40. Jg., 2/2012, S. $271-296$ 
nierten Mitgliederrollen hinaus ein Netzwerk von ad hoc rekrutierten Experten, ${ }^{8}$ um für die routinemäßig stattfindenden Sitzungen seiner beiden Kommissionen Entscheidungen vorzubereiten. Und diese Entscheidungen wirken in vielen Fällen autoritativ, obwohl dem Wissenschaftsrat keine formellen politischen Entscheidungsrechte zustehen. Er ist im Übrigen mit dem erwähnten Abkommen von $1957^{9}$ rechtlich auch nur schwach fundiert, da das Verwaltungsabkommen zwischen Bund und Ländern relativ rasch kündbar ist. Der Wissenschaftsrat besitzt also für bestimmte Themen eine inzwischen als selbstverständlich wahrgenommene institutionelle Autorität, ohne selbst eine robust abgesicherte institutionelle Verfassung zu besitzen. Das erscheint erklärungsbedürftig.

Im Folgenden werden Erklärungen versucht, und dabei stehen nicht die wissenschaftspolitischen, sondern die institutionellen Aspekte des Wissenschaftsrats im Mittelpunkt des Interesses; es geht um Institutionenanalyse. Dafür erscheint als Eröffnungszug Arnold Gehlens Formel von der »Überdeterminiertheit einer dauerhaften Institution « heuristisch instruktiv: Eine solche Institution " muß nicht nur im nächsten, praktischen Sinne zweckmäßig und nützlich sein, sie muß auch Anknüpfungspunkt und >Verhaltens-Unterstützung ‘... höherer Interessen sein «. ${ }^{10}$ Erst dadurch gewinnt sie Reputation, und diese Reputation kann als soziales Kapital auch politisch wirksam sein. Frage dann: In welcher Hinsicht erweist sich der Wissenschaftsrat als zweckmäßig und nützlich? Welchen höheren Interessen dient er auf welche Weise? Wie gelingt ihm der Aufbau von Reputation für sich selber? Und schließlich: Was lernt man, wenn man diesen Fragen nachgeht, über Erfolg und Erfolgsbedingungen von Institutionen, die dem Wissenschaftsrat im Hinblick auf seine institutionellen Besonderheiten in etwa vergleichbar erscheinen? ${ }^{31}$

\section{Institutionelle Rahmenbedingungen}

Die Nützlichkeit einer Institution ist nur in dem sozialen und politischen Kontext bestimmbar, für den sie sich bewähren muss. In dieser Hinsicht ist für den Wissenschaftsrat das hochdifferenzierte Politikfeld entscheidend, in dem er in der Bundes-

8 Der Tätigkeitsbericht 2008 des Wissenschaftsrats weist aus, dass in dem betreffenden Jahr insgesamt 259 externe Sachverständige in Ausschüssen und Arbeitsgruppen zeitweilig mitgewirkt haben, darunter 45 aus dem Ausland (WR 2008 a, S. 677-685). Das übertrifft die Zahl der 32 wissenschaftlichen Mitglieder im Wissenschaftsrat um ein Vielfaches.

9 Verwaltungsabkommen 2008; Röhl 1994, S. 218 ff.; Bartz 2007, S. 266.

10 Gehlen 1957, S. 116.

11 Informativ war für mich die exzellent recherchierte und entsprechend materialreiche Studie über die Geschichte des Wissenschaftsrats von Olaf Bartz (2007). Sehr profitiert habe ich von längeren Gesprächen mit Winfried Benz, Jürgen Gerhards, Max Kaase, Jürgen Kocka, Klaus Lömker, Thomas May, Renate Mayntz, Ulrich Schreiterer, Dieter Simon, Andreas Stucke, Erich Thies und Michael Zürn. Natürlich war nicht folgenlos für meine Einschätzungen, dass ich selber 1980-1987 Mitglied des Wissenschaftsrats und ab 1985 zwei Jahre lang Vorsitzender seiner Wissenschaftlichen Kommission war. 
republik operiert. An der Steuerung der deutschen Wissenschaftspolitik ist eine Vielzahl von kollektiven Akteuren beteiligt, zwischen denen es weit gestreute Kompetenzverteilungen gibt. ${ }^{12}$ Auf der einen Seite wurde die grundgesetzlich verbürgte Garantie der Wissenschaftsfreiheit zugunsten eines relativ hohen Maßes an Autonomie der diversen kleinen und großen Wissenschaftseinrichtungen ausgelegt, in denen Forschung und Lehre stattfinden; der Staat kann in die Funktionsbereiche der universitären und außeruniversitären Organisationen nicht direkt hineindirigieren. Andererseits sind die dem Staat reservierten Steuerungsbefugnisse zwischen dem Bund und den 16 Bundesländern föderalistisch aufgeteilt und zwischen letzteren segmentiert. Diese Umstände erzeugen einen starken Bedarf an integrativen Mechanismen, um den Pluralismus der Wissenschaftspolitik für fällige Innovationen handlungsfähig zu halten. Dabei spielt der Wissenschaftsrat eine wichtige Rolle.

Die Funktion des Wissenschaftsrats wurde bei seiner Gründung 1957 darauf festgelegt, die wissenschaftpolitischen »Pläne des Bundes und der Länder aufeinander abzustimmen «. Obwohl diese starke Vermittlungsformel seit einigen Jahrzehnten in den Verwaltungsabkommen über den Wissenschaftsrat nicht mehr benutzt wird, begründet die Vermittlung von Wissenschaftsinteressen in das Spannungsfeld von Bund und Ländern anhaltend die konstitutive Eigenart des Wissenschaftsrats in dem Maße, in dem ein » kooperativer Föderalismus « das Zusammengehen von Bund und Ländern in wissenschaftspolitisch relevanten Fragen festschreibt. ${ }^{13}$ Ohne den bundesrepublikanischen Föderalismus verlöre der Wissenschaftsrat seine politisch herausragende Funktion.

Die Gegenstände der Vermittlungen, auf die der Wissenschaftsrat im Verwaltungsabkommen festgelegt ist, ergeben sich aus seiner Aufgabe, einerseits "übergreifende Empfehlungen zur inhaltlichen und strukturellen Entwicklung " der Wissenschaft und andererseits - dies auf Anforderung von Bund und/oder Ländern "gutachtliche "Stellungnabmen zu ausgewählten Wissenschaftseinrichtungen zu geben. Er soll einerseits Konzepte über wünschenswerte Wissenschaftsentwicklungen entwerfen und mit den darin postulierten Gütemaßstäben andererseits die Praxis konkreter Wissenschaftsorganisationen kontrollieren. Man könnte das Leitbild für das Wirken des Wissenschaftsrats also darin sehen, dass er im Großen wie im Kleinen Strukturen und Organisationsformen "guter Wissenschaft " entwerfen und überprüfen soll. Es geht zwischen Wissenschaft und Politik im Wissenschaftsrat darum, einen »gemeinsamen Interpretationsrahmen « 14 über die sozialen und poli-

12 Stucke 2010, S. 363 ff.; Braun 1997.

13 Kaase 1999, S. 243 ff., 253 f.; Stucke 2006, S. 250. Die Föderalismusreform hat den »kooperativen Föderalismus « 2006 zwar mit der Folge eingegrenzt, dass der Wissenschaftsrat seine früher maßgeblichen Kompetenzen bei der Regulierung von sogenannten Gemeinschaftsaufgaben von Bund und Ländern teilweise verlor (Bartz 2007, S. 235); gegenwärtig tendiert die Politik aber wieder zur Relativierung der mit der Föderalismusreform ins Grundgesetz hineinformulierten Kooperationsverbote, da die Länder mit ihren Wissenschaftsprogrammen ohne Mitfinanzierung durch Bundesmittel nur unzureichend und überdies in sehr ungleichem Maße handlungsfähig sind.

14 Foemer 1981, S. 83.

Leviathan, 40. Jg., 2/2012 
tischen Bedingungen einer freien, nach ihren eigenen Regeln ausgerichteten und gleichzeitig gesellschaftlich verantwortlich operierenden Wissenschaft herzustellen. Die zentrale » Leitidee « 15 , in der die » höheren Interessen « am Wissenschaftsrat zum Ausdruck kommen, bezieht sich also sowohl auf die Idee einer relativ autonomen Wissenschaft als auch, und das ist das eigentlich Politische seiner Funktion, auf deren Institutionalisierung in einer sich wandelnden Umwelt; darüber soll im Wissenschaftsrat zwischen den Akteuren aus Politik und Wissenschaft ein operationeller Konsens erzielt werden.

Werden in einem solchen Konsens konzeptionell allgemeine und gutachtlich konkrete Stellungnahmen verabschiedet, dann verpflichten sich Bund und Länder nach Artikel 3 des Verwaltungsabkommens nicht nur dazu, dem Wissenschaftsrat für seine Zwecke Auskunft zu geben, sondern sie sichern seinen Empfehlungen überdies zu, sie »bei der Aufstellung ihrer Haushaltspläne im Rahmen der haushaltsmäßigen Möglichkeiten zu berücksichtigen «. Mit diesem förmlichen Berücksichtigungsversprechen gewinnt der Wissenschaftsrat gegenüber der großen Menge bestehender Politikberatungsgremien einen politischen Geltungsvorsprung; er betreibt nicht nur die Beratung von Politik, sondern auch Vorabstimmungen zwischen Wissenschaft und Politik. Solche Vorabstimmungen sind für die politischen Entscheidungsinstanzen nicht formell verbindlich - und sie wurden von Fall zu Fall tatsächlich auch nur in sehr ungleichem Maße umgesetzt; aber den vom Wissenschaftsrat verabschiedeten Empfehlungen ist das Privileg verliehen, wenigstens "berücksichtigt « zu werden. Das ist für Politikberatung keineswegs der Normalfall.

Der politischen Sonderstellung des Wissenschaftsrats entspricht, dass auch seine Mitgliederzusammensetzung eigenartig ist. ${ }^{16} \mathrm{Er}$ stellt sich als ein sorgfältig balanciertes Gremium aus Politik und Wissenschaft dar. Die Politik ist, mit gleich gewichteter Stimmenzahl ${ }^{17}$ von Bund und Ländern, im Wissenschaftsrat selber vertreten, bleibt also nicht nur Adressat seiner Empfehlungen. Dem Verwaltungsrat, den Bund und Länder im Wissenschaftsrat bilden, stehen mit einem insgesamt gleichen Stimmengewicht die ehrenamtlich tätigen 32 Mitglieder der Wissenschaftlichen Kommission gegenüber. Sie besitzen einen als politisch unabhängig symbolisierten Status dadurch, dass sie auf Zeit vom Bundespräsidenten berufen werden. Diesem werden sie mehrheitlich von der sogenannten Allianz, dem Verbund der großen Wissenschaftsorganisationen, vorgeschlagen, ${ }^{18}$ stellen also keine demokratisch vermittelte Repräsentation der Wissenschaft und der Interessenverbände von

\section{Hauriou 1965, S. 36.}

16 Benz 1996, S. 1670 ff.; Stucke 2011, S. 160 ff.

17 Der Bund hat genauso viele Stimmen wie die Summe der Länder, nämlich 16, und die Mitgliederzahl der Wissenschaftlichen Kommission gleicht mit 32 der Summe der Stimmen von Bund und Ländern.

18 Der Allianz gehören nach der Erweiterung des Gremiums an: der Wissenschaftsrat selbst sowie die Deutsche Forschungsgemeinschaft, die Max-Planck-Gesellschaft, die Helmholtz-Gemeinschaft, die Leibniz-Gemeinschaft, die Fraunhofer-Gesellschaft, die Deutsche Rektorenkonferenz, die Leopoldina als Repräsentanz der deutschen Akademien, die Humboldt-Stiftung sowie der Deutsche Akademische Austausch-Dienst (DAAD). 
Professoren und Assistenten dar. ${ }^{19}$ Die Mitglieder der Wissenschaftlichen Kommission gehören in ihrer Mehrheit zum Establishment der in der Wissenschaft und ihrer Selbstverwaltung bewährten Kollegen und Kolleginnen.

Die Meinungsbildung über anstehende Konzepte und Gutachten vollzieht sich im Wissenschaftsrat zuerst in ad hoc berufenen Expertenausschüssen, sodann in den beiden Kommissionen und am Ende in der Vollversammlung, wobei laut Verwaltungsabkommen die Beschlüsse der Vollversammlung jeweils »von der Wissenschaftlichen Kommission unter fachlichen und wissenschaftlichen Gesichtspunkten und von der Verwaltungskommission unter verwaltungsmäßigen und finanziellen Gesichtspunkten vorbereitet " werden sollen. ${ }^{20}$ Die ersten Schritte vollziehen sich zumeist in der Regie der Wissenschaftlichen Kommission. Die in der Wissenschaftskommission formulierten Wünschbarkeiten stoßen im Fortgang auf das juristisch und finanziell gebundene Realitätsprinzip der Verwaltungskommission, bevor beide zusammen in der Vollversammlung entscheidungsfähig werden. Hierbei ist für Betrieb und Wirkung des Wissenschaftsrats nun entscheidend, dass seine Beschlüsse nicht nur in der Vollversammlung, sondern auch in den beiden Kommissionen " mit einer Mehrheit von zwei Dritteln der abgegebenen Stimmen " getroffen werden müssen. Dies setzt ihn unter einen außergewöhnlich starken Konsensdruck. Dieser Konsensdruck bestimmt die Interaktionen von Politik und Wissenschaft im Wissenschaftsrat auf eine elementare Weise: Beide müssen, um ihre Interessen durchsetzen zu können, miteinander reden - und zwar so, dass sie einander überzeugen, weil kraftvolle Druckmittel im Wissenschaftsrat eher innerhalb der Verwaltungskommission als zwischen Verwaltungskommission und Wissenschaftlicher Kommission verfügbar sind. Gelingt dies nicht, kommen keine Entscheidungen zustande - oder aber diese werden mit rhetorischen Leerformeln immunisiert. Gelingt es aber, und das ist oft der Fall, wird auch der im Hintergrund bleibende, gleichwohl bedeutsame Einfluss der Geschäftsstelle des Wissenschaftsrats in aller Regel eine Rolle gespielt haben; darauf wird zurückzukommen sein.

19 Das Demokratiedefizit führte besonders in den späten 1960er Jahren zu Zweifeln an der politischen Legitimität des Wissenschaftsrats (Bartz 2007, S. 113 ff.; Röhl 1994, S. $164 \mathrm{ff}$.), die ihn allerdings nicht anhaltend beschädigten.

20 Das Plädoyer Ernst-Ludig Winnackers zur Abschaffung des Wissenschaftsrats wird mit der Vorstellung verbunden, an seiner Stelle »Ad hoc-Kommissionen « mit Besetzungsvorschlägen des DFG-Präsidenten einzurichten. Angesichts des breiten Spektrums von Tätigkeiten, die der Wissenschaftsrat übernommen hat, müsste es sich dabei um eine beachtliche Zahl von Kommissionen handeln. Um deren Vorschläge für eine in sich stimmige Wissenschaftspolitik aufeinander abstimmen zu können, bedürfte es einer koordinierenden Dachorganisation. Mit deren Einrichtung wäre man aber wieder bei einer Art Wissenschaftsrat. Dies umso mehr, wenn man mehr erreichen will als bloße Beratung. Der institutionelle Mehrwert des Wissenschaftsrats ergibt sich daraus, dass er zwischen Wissenschaft und Politik Vorabstimmungen über anstehende politische Entscheidungen zu treffen sucht. Der damit verbunden hohe Konsensbedarf macht ihn einerseits schwerfälliger und langsamer, andererseits aber auch politisch verbindlicher - wenn es gelingt, diesen Konsens zu erreichen. 
Theoretisiert man diese Besonderheiten, so fällt die Vergleichbarkeit des Wissenschaftsrats mit den verfassunggebenden Versammlungen auf, die Jon Elster mit dem Konzept von »deliberative setting " zuerst an historischen Beispielen, nach dem Zerfall des sowjetischen Imperiums auch in osteuropäischen Ländern untersucht hat: ${ }^{21} »$ Deliberation about constitutions requires the creation of what I called a deliberative setting. The procedure must ... allow for communicative interaction. Also, the setting must steere this interaction toward arguing and away from threatbased bargaining «. ${ }^{22}$ In Wirklichkeit findet man zwar immer eine Mischung aus »arguing and bargaining " in solchen Kommunikationen, aber: »Pure argument is observed, or is at least supposed to be the rule, in juries, in which unanimity is required $« .{ }^{23}$ Es gilt für diesen Kontext das Diskursprinzip mit dem »Vorrang des Arguments vor dem Interesse am Argument «. ${ }^{24}$ Das heißt nicht, dass sich die Interessenmotive dort ins Nichts auflösten; sie bleiben im Hintergrund natürlich bedeutsam. Aber das Interesse muss sich im »deliberative setting " der Beratungen des Arguments bedienen, um hier Geltung zu erlangen - und das wird für das Handeln kaum völlig folgenlos bleiben.

Der Wissenschaftsrat lässt sich als »deliberative setting " in diesem Sinne begreifen. Er ist - dies auch im Sinne von Habermas ${ }^{25}$ - zumindest von seiner institutionellen Anlage her ein im politischen Raum seltenes Beispiel für das Arrangement von Diskursen - neben allem Bedarf an pragmatischen Verhandlungen und oberhalb der politischen Interessen, die auch in diesem Setting immer mit im Spiel sind und die sich spätestens bei den Entscheidungsformulierungen dann auch geltend machen. Der Wissenschaftsrat ist mit seinem institutionellen Zuschnitt kein unabhängiger Think Tank, der in Einsamkeit und Freiheit vor sich hinräsonieren könnte. Er ist eine politische Institution, und deshalb kann nicht überraschen, dass seine Texte ein Amalgam reflexiver Auseinandersetzungen und politischer Kalkulationen darstellen. Bei deren Mischung aber erweist sich der Wissenschaftsrat, so ein Beobachter von der Bank der Verwaltungskommission, als ein »Ort relativ machtfreien Sprechens ${ }^{2}{ }^{26}$

21 Elster 1986, 1989 a, 1989 b.

22 Elster 1989 b, S. 105.

23 Elster 1989 a, S. 6 f.

24 Siehe van den Daele 1996, S. 302.

25 Habermas 1992, S. 410 ff.

26 Gleichwohl merkt dieser Ministerialbeamte auch für die von ihm gerühmte Sprechkultur der Wissenschaftlichen Kommission an: "Dass Wissenschaftler untereinander durchaus fähig sind, in solchen Beratungen Minderheitsmeinungen unterzubuttern und gar zu mobben, habe ich nicht nur hier, sondern auch in der DFG mit Vergnügen festgestellt. Die Underdogrolle der Fachhochschulvertreter ist ein Thema für sich «. 


\section{Spannungen und Entspannungen}

Institutionelle Arrangements können nicht hinreichend erklären, was in Institutionen tatsächlich geschieht. Institutionen entstehen nicht nur aufgrund der Bedingungen, die sie möglich machen. Sie eröffnen Spielräume für soziales Handeln. Sie sorgen dafür, dass "Anlässe für die Interaktion bestimmter Akteure definiert und Arenen geschaffen werden, in denen spezifizierte Akteure zur Beratung oder Entscheidung über spezifizierte Themen zusammenkommen, wobei sie bestimmten Entscheidungsregeln unterworfen sind «. Aber: »Für die Nutzung der faktisch verbleibenden Handlungsspielräume sind die jeweiligen Handlungsorientierungen der Akteure von ausschlaggebender Bedeutung ${ }^{.27}$ Erst die Akteurszentrierung eines institutionalistischen Erklärungsprogramms sichert diesem seine Gültigkeit.

Entsprechend bleibt dann auch für den Wissenschaftsrat zu fragen, wie seine Mitglieder sowohl mit den Freiheitsgraden als auch mit den Restriktionen umgehen, die sich aus ihrem institutionellen Kontext ergeben. Wie lösen die Akteure die zwischen ihnen vorhandenen Interessenspannungen? Stellen sich die Interaktionen miteinander wirklich als »deliberativ « dar? Gelingen auf diese Weise Verständigungen? Im Hinblick auf was gelingen sie nicht? Und welche Bedingungen entscheiden über das eine oder das andere?

Der Ausgangspunkt aller Interaktionsdynamiken liegt im Wissenschaftsrat darin, dass er ein Gemisch sowohl gemeinsamer als auch einander widersprechender Interessen verkörpert. Konsensbildung ist unter diesen Bedingungen möglich, aber oft schwierig. Sie ist oft schwierig, weil die Spannungslinien, die der Verständigung der Akteure entgegenstehen, sowohl innerhalb der Politik als auch zwischen Politik und Wissenschaft strukturell angelegt sind. Sie ergeben sich im Verhältnis zwischen Wissenschaft und Politik dadurch, dass die Wissenschaft im Kampf um ihre Autonomie für sich Spielräume und Ressourcen beansprucht, welche den herrschenden Interessen zuwiderlaufen und die Möglichkeiten der Politik häufig überfordern. Hinzu kommen Interessenkonflikte im Politikbereich sowohl zwischen den Ländern, die miteinander konkurrieren, als auch zwischen ihnen und dem Bund. Letzterer wünscht sich Verfügungsrechte, die im Kulturföderalismus der Bundesrepublik den Ländern verliehen sind; diese benötigen umgekehrt vom Bund Geld, für das dieser nun aber auch einen Verfügungsgewinn erwartet.

In den Sitzungen des Wissenschaftsrats machen sich die Interessenspannungen zwischen den Akteursgruppen gelegentlich mit offenen Kontroversen bemerkbar. ${ }^{28}$ Vermieden bzw. stillgestellt werden diese mit zwei Strategien, welche die Operationsfähigkeit des Wissenschaftsrats und seinen Zusammenhalt sichern. Ent-

27 Mayntz, Scharpf 1995, S. 48, S. 52.

28 In den Sitzungsprotokollen, für deren Entwurf die Geschäftsstelle des Wissenschaftsrats verantwortlich ist, kommen diese Kontroversen kaum zum Ausdruck. Sitzungsprotokolle erfüllen ihre Funktionen in Organisationen, die auf Konsens angelegt sind, durch windschnittig zubereitete Berichterstattungen vornehmlich über das, was aus Sitzungen an greifbaren Entscheidungen herausgekommen ist. Die schriftliche Markierung von Fronten führt eher zu deren Versteifung. 
scheidend im Hinblick auf Entspannungsstrategien ist erstens eine Agendapolitik, mit der in den jährlichen Arbeitsprogrammen des Wissenschaftsrats aufgrund von Vorlagen der Geschäftsstelle und der hinter ihnen stehenden Absprachen zwischen den Vorsitzenden und dem Generalsekretär solche Themen unter den Tisch fallen, zu denen angesichts tief reichender politischer Konflikte Verständigungen zwischen Wissenschaftlicher Kommission und Verwaltungskommission - nicht zuletzt aber auch innerhalb der Verwaltungskommission - von vornherein ausgeschlossen erscheinen. »Der Wissenschaftsrat hat, wie jeder, der ihn kennt, weiß, nie eine große Neigung gezeigt, die politisch wirklich heißen Themen anzufassen. Seine Sache waren vorzugsweise ... die gemäßigt kontroversen Themen « - so ein ehemaliger Vorsitzender der Wissenschaftlichen Kommission..$^{29}$ Die Erfolge des Wissenschaftsrats, von denen später die Rede sein wird, sind insoweit begleitet von einer Serie ausgeschlossener Themen, die das Gremium sich nicht getraut hat zu bearbeiten. Oder aber - das ist neben seinen agendapolitischen Ausschließungstaktiken die gewissermaßen sprachpolitische Dimension seiner internen Verständigungsbemühungen der Wissenschaftsrat hat Themen, die nicht zu verdrängen waren, mit Kompromissformeln offen gehalten, bei denen vor allem durch den Gebrauch des Konjunktivs und mit "Sowohl-als-auch «-Konstruktionen Unverbindlichkeit ausgedrückt wurde. ${ }^{30}$ Das integrative Geschick vor allem der Geschäftsstelle des Wissenschaftsrats, die für die Formulierung nicht nur der Protokolle, sondern auch der meisten Entscheidungsvorlagen sorgt, bewährte sich nicht zuletzt durch rhetorisch kunstvolle Nutzungen dessen, was in der Wissenschaftstheorie als "pseudo-normative Formel « benannt ist. ${ }^{31}$ Ihr Charakter als »Quasi-Leerformel « hat die Funktion, einen Dissens zu verbergen, ohne das Thema, auf das er sich bezieht, völlig zu beschweigen.

29 Kielmansegg 1998.

30 Bei den Texten des Wissenschaftsrats fällt nicht nur die immer wieder geschmeidige Pflege des Ungefähren auf, sondern auch das hohe Maß an Selbstreferenz. Unterzieht man diese Texte einer Zitationsanalyse, so fällt bei dem Vergleich von verschiedenen Jahrzehnten seiner Tätigkeit nicht nur eine deutliche Zunahme von Quellenhinweisen insgesamt (also gewissermaßen eine Akademisierung seiner Textproduktion), sondern auch eine anhaltend hohe Selbstzitationsrate auf. Immer wieder erinnert der Wissenschaftsrat seine Leser daran, dass er das, was jetzt zu sagen ist, auch früher schon gesagt hat. Ist dies, so zeigt eine Überprüfung, zwar durchweg richtig, frappiert gleichwohl der rechthaberische Gestus ständiger Selbstzitation; er folgt eher den Mustern politischer als akademischer Rhetorik.

31 Degenkolbe 1965, S. 330 ff. Zwei Textbeispiele für diskrete Einlassungen aus einem Evaluationsbericht (Drs. 10465-11, 47,50): »Der Wissenschaftsrat hielte es deshalb für sinnvoll ..." ", etwas Bestimmtes zu tun - oder eine Leistung sei »... aus Sicht des Wissenschaftsrats noch steigerungsfähig ...«. Ein interessantes Beispiel von » Formulierungstaktik « berichtet auch Foemer (1981, S. 138 ff.). 
Immunisierungstaktiken sowohl der Dissensvermeidung als auch der Dissensverschleierung lassen sich an vielen Beispielen illustrieren. ${ }^{32}$ Solche Beispiele machen die Grenzen deutlich, in denen der Wissenschaftsrat operiert. Er kann einen politischen Dissens, der in ihn hineingetragen wird, mit Erfolgsaussicht nur bearbeiten, wenn ihn die Parteipolitik, die in der Verwaltungskommission auf der Bundes- wie auf der Länderbank repräsentiert ist, nicht fundamentalisiert hat. Spielräume für seine Vermittlungen findet er nur in dem Maße, in dem das politische System die politischen Positionen nicht polarisiert. Das ist zum Vorteil einer Einrichtung wie des Wissenschaftsrats in dem stark in sich verflochtenen Politiksystem der Bundesrepublik nur begrenzt der Fall, stellt andererseits aber auch keine Ausnahme dar. Wo der Wissenschaftsrat bei der Einschätzung seiner Möglichkeiten vorab jeweils deren Grenzen sieht, wird im Einzelfall oft strittig sein. Aber in einem demokratischen Gemeinwesen mit konstitutioneller Gewaltenteilung wird eine demokratisch nicht ausdrücklich zu politischen Entscheidungen legitimierte Institution schon aus Selbsterhaltungsinteresse gut daran tun, ein Prinzip von "political self-restraint « 33 zu beachten, um nicht ins Abseits zu geraten.

Die Beschränkung seiner Themen und die fallweise Verunklarung von Entscheidungen durch Unverbindlichkeiten gehören zu den Kosten, die der Wissenschaftsrat entrichtet, um politisch handlungsfähig zu bleiben. Ob das vom Wissenschaftsrat immer richtig disponiert ist, lässt sich nicht prinzipiell beurteilen. Die notwendige Balance zwischen Indifferenz und Engagement im Hinblick auf die für ihn einschlägigen Themen erscheint als ein Dauerproblem, dessen jeweilige Lösungen strittig

32 Zum Beispiel: Ausmaß und Gründe der eklatanten Unterfinanzierung deutscher Hochschulen wurden in Stellungnahmen des Wissenschaftsrats zwar immer wieder mal angesprochen, aber nicht ständig so skandalisiert, wie es angebracht gewesen wäre; die Wissenschaftsinteressen an einer liberalen Fassung von Datenschutzbestimmungen, welche die Wissenschaftliche Kommission vertrat, fand keine Anerkennung in der Verwaltungskommission, kamen in den Entscheidungen also auch nicht zur Sprache; das Thema der Studiengebühren wurde angesichts der dazu gespaltenen Politik im Wissenschaftsrat in den Rang einer einzigen Fußnote degradiert, in der zur Sache nichts gesagt wurde; früh schon wurde die Vorstellung einer Ablösung des Abiturs als Studienzulassungsvoraussetzung vor allem aufgrund entgegenstehender Länderinteressen fallengelassen und nicht wieder aufgenommen; und bei allen Einlassungen zu Entwicklungen der Hochschulverfassungen war der Wissenschaftsrat peinlich darauf bedacht, zur Mitbestimmungsfrage in den Hochschulgremien unanstößig zu bleiben - es war dem Bundesverfassungsgericht vorbehalten, dazu relativ spät eindeutig zu werden. Vgl. Bartz 2007, S. 186 ff.; Benz 1996, S. 1686; Kaase 1999, S. 249 f.; Kielmansegg 1990, S. 214 ff.; Stucke 2011, S. 168 f.

33 Das Selbstbeschränkungsprinzip ist mit Blick auf das Funktionieren von Gewaltenteilung zuerst als Prinzip des »judicial self-restraint « der Verfassungsjustiz postuliert worden. Es lässt sich aber wohl im Hinblick auf andere Institutionen, die im politischen Raum operieren, also auch für den Fall des Wissenschaftsrats, im Sinne einer Definition des Bundesverfassungsgerichts (BVerfG 36,1 S. 14 f.) verallgemeinern: »Der Grundsatz des judicial restraint, den sich das Bundesverfassungsgericht auferlegt, bedeutet nicht eine Verkürzung oder Abschwächung seiner ... Kompetenz, sondern der Verzicht >Politik zu treiben , d.h. in den von der Verfassung geschaffenen und begrenzten Raum freier politischer Gestaltung einzugreifen «.

Leviathan, 40. Jg., 2/2012 
und für den Zusammenhalt der Institution selber heikel sind; sie sind heikel vor allem für das Verhältnis von Wissenschaftlicher Kommission und Verwaltungskommission, weil der Wissenschaftsrat, bevor er nach außen wirksam wird, immer auch seine eigenen Mitglieder überzeugt haben muss. Und dazu gehört das Bedienen nicht nur politischer Interessen, sondern auch der Mitwirkungsmotivationen jener ehrenamtlich tätigen Wissenschaftler, auf deren zeitraubenden Einsatz er setzen muss. ${ }^{34}$ Ein Übermaß an Ausblendung wissenschaftspolitisch drängender Themen kann nicht im Sinne engagierter Mitglieder der Wissenschaftlichen Kommission sein; sie verlieren dann die Lust mitzumachen.

\section{Kommunikations- und Entscheidungsprozesse}

Anscheinend ist die angesprochene Balance im Wissenschaftsrat bislang insoweit gelungen, als die damit verbundenen Spannungen nicht zu seinem Ende oder zu einem Stillstand seiner Arbeiten geführt haben. ${ }^{35}$ Das hat damit zu tun, dass die Interessenspannungen von der Gemeinsamkeit des Interesses am Funktionieren des Wissenschaftsbetriebs selbst überlagert und gedämpft werden. Jenseits aller Differenzen liegt das Gedeihen von Wissenschaft letztlich im Interesse aller, die sich in dieser Arena versammeln.

Zwei Umstände kräftigen dieses kollektive Interesse, und beide haben mit der Gemeinsamkeit bestimmter externer Bezugsgruppen zu tun, gegen die sich Wissenschaft und Wissenschaftspolitik gleichermaßen behaupten müssen. Erstens ist die im Wissenschaftsrat vertretene Politik eine Sache von Wissenschaftspolitikern, die sich in den politischen Entscheidungsgremien von Bund und Ländern ständig gegen außerwissenschaftliche Ressortinteressen durchzusetzen haben; dass ihnen dies einigermaßen gelingt, ist Bedingung ihres eigenen politischen Erfolgs. Gegenüber ihren Finanzministern sind die Mitglieder der Verwaltungskommission auf der Seite der Wissenschaft. Hinzu kommt weiterhin der gemeinsam empfundene Druck, der sich aus der Internationalisierung der Wissenschaft für die deutsche Wissenschaftspolitik ergibt. Nicht zufällig ist im Verwaltungsabkommen zwischen Bund und Ländern im Jahre 2008 dem Wissenschaftsrat verordnet worden, er habe "zur Sicherung der internationalen Konkurrenzfähigkeit der Wissenschaft in Deutsch-

34 Kaase 1999, S. 252.

35 Es gibt nur einen bekannt gewordenen Fall, bei dem ein Mitglied der Wissenschaftlichen Kommission vorzeitig aus dem Wissenschaftsrat ausgeschieden ist, um diesen anschließend auch öffentlich zu kritisieren; das passierte in den 1970er Jahren (Bartz 2007, S. 269). Auch die Zahl der Fälle, in denen Wissenschaftler eine zweite Amtszeit im Wissenschaftsrat ausschlugen, ist insgesamt sehr gering - was umso bemerkenswerter deshalb ist, als die Beanspruchung vor allem der Wissenschaftlichen Kommission durch die Arbeiten des Wissenschaftsrats in der Regel sehr hoch ist. - Von der Geschäftstelle wird berichtet, dass auch die Bereitschaft externer Experten, an Ausschüssen und Bewertungsgruppen des Wissenschaftsrats unhonoriert mitzuwirken, unverändert hoch sei; es gäbe keine Rekrutierungsprobleme (vgl. Fußnote 18). 
land ... beizutragen «. Die Berufung auf nationale Interessen ist in beiden Kommissionen des Wissenschaftsrats immer gut für starke Argumente.

Beide Umstände befördern das wechselseitige Interesse der Mitglieder von Verwaltungs- und Wissenschaftlicher Kommission aneinander. »Offensichtlich hat sich die professionelle Dignität der Wissenschaftlichen Kommission für den Bund wie für die Länder als ein äußerst wirksames Legitimationsinstrument erwiesen, mit dem eventuell Konflikte externalisiert und über Reputation neutralisiert werden können. ${ }^{36}$ Man kann sich in den Parteien und in den Ministerialverwaltungen manches politische Argument mit dem Hinweis ersparen, der Wissenschaftsrat habe dies und das schon festgelegt. Dies konnte zum Beispiel auch im großen Maßstab funktionieren, als der Wissenschaftsrat und vor allem die Wissenschaftliche Kommission bei der Vereinigung der Wissenschaftssysteme von BRD und DDR am Beginn der 1990er Jahre die in zentralen Bereichen auch politisch folgenreiche Regie übernahm und in einem beispiellosen Einsatz eine Assimilation der Ost- in die Westwissenschaften betrieb. ${ }^{37}$ Die Autorität des Wissenschaftsrats hat hier, aber auch in vielen anderen Fällen die politische Brisanz der Entscheidungen stillgestellt. ${ }^{38}$ Umgekehrt konnten solche Erfahrungen gerade auch dem Bedeutungsanspruch der wissenschaftlichen Mitglieder des Wissenschaftsrats konvenieren. Dieser vermittelt ihnen über den persönlichen Zugang zu den wissenschaftspolitisch maßgeblichen Akteuren des Landes einen Einfluss, den sie in den üblichen Gremien der Politikberatung nicht erfahren könnten. Insofern löschen auch Frustrationen über die Grenzen dieses Einflusses nicht die Erfahrung, dass es diesen gibt.

In der Austauschlogik rationaler Akteure stellt die Reziprozität ihrer Leistungen füreinander eine zentrale Voraussetzung dafür dar, dass der Wissenschaftsrat auch in den von ihm nicht lösbaren Konflikten eine Notlösung finden kann, »to agree not to agree «. Der Umgang miteinander bleibt in seinen Sitzungen grundsätzlich unpolemisch. Vor allem die Mitglieder der Verwaltungskommission, gewöhnt an die nicht selten kruden Geräuschentwicklungen in rein politischen Gremien (manchmal eben auch bei Kultusministerkonferenz und Gemeinsamer Wissenschaftskonferenz) erleben den Umgangsstil im Wissenschaftsrat, so hört man, als »ausgesprochen höflich " und seine Diskussionen als "erholsam sachlich «, nicht selten wohl auch als intellektuell anregend. Die Gründe dafür liegen vornehmlich darin, dass der Wissenschaftsrat in seinem Betrieb ein prozedurales Gleichgewicht von Wissenschaft und Politik herstellt und dass dabei die Wissenschaftskommission des Wissenschaftsrats gegenüber den politischen Konfliktlagen von Bund und Ländern

36 Kaase 1999, S. 247; ähnlich Röhl 1994, S. 155.

37 Simon 1991; Bartz 2007, S. 158-183.

38 Und diese Leistung hat den politischen Finanziers des Wissenschaftsrats nur wenig Geld gekostet. Auch darüber hinaus ist die vielfältige Konzeptions- und Evaluationsleistung vor allem der Wissenschaftlichen Kommission für die Politik angesichts der durchweg unentgeltlich erbrachten Leistungen der Mitglieder und der von ihnen zahlreich kooptierten Experten außerordentlich preisgünstig zu haben. Seine Effektivität, könnte man sagen, ist sehr effizient. Dies auch mit Blick darauf, dass die Finanzausstattung der Geschäftsstelle sehr knapp gehalten wird. 
die im Sinne Georg Simmels zivilisierende Rolle eines Dritten spielt: Vor einem Dritten muss sich jede Partei »objektiver äußern ... als bei unmittelbarem Gegenüberstehen. Denn es muß ihr jetzt darauf ankommen, auch den Vermittler für ihren Standpunkt zu gewinnnen ... « ${ }^{39}$ Die Wissenschaftler im Wissenschaftsrat stellen für die Verwaltungskommission ein Publikum dar, bei dem sich mit politischer Agitation nicht punkten lässt; die Konsensbedingungen des Wissenschaftsrats bringen aber mit sich, dass man bei ihnen punkten muss, wenn man etwas erreichen will.

Für die friedlichen Diskussionsstile des Wissenschaftsrats und seiner diversen Gremien sind bei den Mitgliedern der Wissenschaftlichen Kommission einige Voraussetzungen strukturell angelegt. Für ein gewisses Maß an Erfahrung im Umgang mit den pragmatischen Zwängen praktischen Handelns ist schon durch die Praxis ihrer Rekrutierung gesorgt. Es handelt sich bei ihnen weder um Wissenschaftsfunktionäre, die einem der Interessenverbände der Wissenschaft rechenschaftspflichtig sind und sich angesichts dieser Bindung gedrängt fühlen müssen, im Konfliktfall Argumentation durch Verlautbarung zu ersetzen, noch um akademische Wissenschaftsforscher mit vornehmlich theoretischen Interessen. ${ }^{40}$ Meistens sind die Mitglieder der Wissenschaftlichen Kommission ehemalige Amtsträger der wissenschaftlichen Selbstverwaltung - zum Beispiel Dekane und Rektoren, Institutsdirektoren und Gutachter der Deutschen Forschungsgemeinschaft -, welche bei der Vorauswahl durch die sogenannte "Allianz « und bei der Ernennung durch den Bundespräsidenten als praktisch bewährt, als wissenschaftlich reputierlich und auch als persönlich interessant angesehen werden können. Zweifel an der demokratischen Legitimität eines derart rekrutierten Gremiums konnten, als sie Ende der 1960er Jahre aufkamen, wieder zurücktreten, da es bei den Funktionen des Wissenschaftsrats nicht um die unmittelbare Herstellung politischer Entscheidungen, sondern um revidierbare Vorabstimmungen dazu geht. Der Vorteil der Rekrutierungspraxis der Wissenschaftlichen Kommission liegt andererseits darin, dass mit dem gewählten Verfahren neben einem Mindestmaß an wissenschaftspolitischer Erfahrung auch ein Potential an praktischem Augenmaß und an Kompromissfähigkeit gewonnen wird,

39 Simmel 1968, S. 77. Die Rolle eines unabhängigen Dritten wird dadurch symbolisiert, dass die Ernennung der Mitglieder der Wissenschaftlichen Kommission nicht von der exekutiven Politik, der sie ja die Ergebnisse vermitteln soll, sondern vom Bundespräsidenten vorgenommen wird. In dessen Haushalt ist auch die hochrangige Ministerialdirektorenstelle des Generalsekretärs des Wissenschaftsrats eingestellt. Als dies im November 1988 auf Initiative der Verwaltungskommission geändert werden sollte, kam es zu einem Streik der Wissenschaftlichen Kommission: Sie zog aus der Vollversammlung aus und machte diese damit beschlussunfähig. In einer außerordentlichen Vollversammlung kassierte die Verwaltungskommission ihre Pläne umgehend, und es blieb beim alten Status quo (Bartz 2007, S. 267 f.).

40 Mit Recht spricht Andreas Stucke deshalb davon, dass der Wissenschaftsrat »not a body for scientific policy advice, but one for policy advice by scientists « darstelle (Stucke 2011, S. 161). 
das den Erörterungen im Wissenschaftsrat positionelle Flexibilität und am Ende auch Entscheidungsfähigkeit sichern kann. ${ }^{41}$

Auch diese Besonderheiten haben dazu geführt, dass der Wissenschaftsrat innerhalb seiner von außen vorgegebenen, aber ebenso selbstbegrenzten thematischen Spielräume produktiv werden konnte. Er stellt sich, was sein Innenleben angeht, als ein relativ erfolgreicher Selbstverständigungsbetrieb dar. Die Beschlussfähigkeit seiner Vollversammlungen drückt sich Jahr für Jahr in einer großen Zahl von Konsensentscheidungen aus. Über die Jahrzehnte hin ist die Zahl der allgemeinen konzeptionellen Empfehlungen über Stand und Entwicklung des deutschen Wissenschaftssystems zwar in etwa gleich geblieben, zeitweilig sogar rückläufig gewesen, aber die Zahl der gutachterlichen Urteilstexte über universitäre und vor allem außeruniversitäre Lehr- und Forschungseinrichtungen hat sehr deutlich zugenommen. ${ }^{42}$ Die Frage ist, ob und in welcher Weise diese dann auch externe Resonanz erzeugt haben; das ist das entscheidende Erfolgskriterium für sein Funktionieren.

\section{Wissenschaftspolitische Resonanzen}

Auch wenn im Wissenschaftsrat zu einer Entscheidungsvorlage Konsens entstanden ist, lässt sich die damit verabschiedete Empfehlung nicht nach Linearitätsmustern im Ein-zu-Eins-Verhältnis in die Praxis umsetzen. Mit dem Verlassen der Arena des Wissenschaftsrats treten diese Empfehlungen aus einem »deliberativen Setting « heraus und geraten in den politischen Handlungsbereich, der nach anderen Regeln funktioniert. Und für einen reibungslosen Transfer sorgt nicht schon, dass die wissenschaftspolitisch verantwortlichen Akteure beim Konsens im Wissenschaftsrat mitgemacht haben. Die juristische Formel von der »Verpflichtung aus vorausgegangenem Tun ${ }^{43}$ greift hier nur bedingt. Die Selbstbindung an die im Wissenschaftsrat eingenommenen Positionen wird bei Wissenschaftspolitikern und Administratoren zwar so weit reichen, dass Hemmungen existieren, außerhalb des Wis-

41 Dies gilt auch für die Rekrutierung der großen Zahl von externen Experten in Ausschüsse und Bewertungsgruppen des Wissenschaftsrats. Die informelle Regel ist: Es werden keine Interessenvertreter von Interessengruppen gewählt, die im Akteursfeld der Wissenschaftspolitik operieren.

42 Der jährliche Ausstoß des Wissenschaftsrats hat sich in den letzten 30 Jahren in etwa vervierfacht. Anfang der 1980er Jahre lagen die veröffentlichten Empfehlungen und Gutachten bei durchschnittlich 10, Anfang der 1990er Jahre bei ungefähr 20-25, 1999/2000 bei knapp 40 und 2009/2010 bei mehr als 40. Dabei hat sich das relative Gewicht weg von allgemeinen Stellungnahmen hin zu speziellen Evaluationen von Forschungseinrichtungen und sogenannten Akkreditierungen von Hochschuleinrichtungen verschoben. Auch im Wissenschaftsrat wird immer wieder darüber diskutiert, ob die Überbeanspruchung nicht auf Kosten der Qualität der beschlossenen Texte geht. Er sieht sich hier in dem notorischen Bewährungsdilemma: Als je erfolgreicher eine Einrichtung wahrgenommen wird, umso mehr mutet man ihr zu (ein Dilemma deshalb, weil sich die nachfolgenden Belastungseffekte seines Erfolgs von irgendeinem Punkt an als Bedingung für das Verschwinden des Erfolgs erweisen können).

43 Kielmansegg 1990, S. 217.

Leviathan, 40. Jg., 2/2012 
senschaftsrats völlig entgegengesetzte Positionen zu vertreten; die Akteure würden, täten sie es, im Wissenschaftsrat selber diskreditierbar sein, und daran kann ihnen nicht liegen, solange sie den Wissenschaftsrat für andere Zwecke noch zu brauchen glauben. Aber es sind jenseits eines eklatanten Selbstwiderspruchs gesichtswahrende Anpassungsstrategien verfügbar, um politischen Opportunitäten genügen zu können. Es gibt, und dafür lässt sich schon bei den Entscheidungsformulierungen im Wissenschaftsrat sorgen, nutzbare Deutungsspielräume bei uneindeutig bleibenden Festlegungen. Es gibt für angemahnte Politikvollzüge Vertagungschancen, verbindbar mit Absichtserklärungen, die sich nicht sofort reklamieren lassen. Es gibt nicht zuletzt die Vorteile eines kasuistischen Rekurses auf besondere Umstände und die Behauptung eines Zwangs zur Ausnahme. Der Wissenschaftsrat würde jedoch seinen politischen Sinn verlieren, wären solche politischen Ausweichmanöver die wahrnehmbare Regel. Es lässt sich deshalb auch zeigen, dass er mit seinen Setzungen in der wissenschaftspolitischen Praxis immer wieder eine nicht unbedeutende Rolle spielt.

Der Wissenschaftsrat wirkt auf zweifache Weise auf die Wissenschaftspolitik der Bundesrepublik ein. Erstens - und für seine Reputation grundlegend - durch richtungsweisende Empfehlungen und zweitens durch institutionelle Evaluationen, deren Standards aus den programmatischen Stellungnahmen begründbar erscheinen. Misst man (1) den Effekt der konzeptionellen Empfehlungen für die Entwicklung des deutschen Wissenschaftssystems an einem Maßstab, der in der Wissenschaft selber üblich geworden ist, nämlich an den Zitationen, die sich in der wissenschaftspolitischen Literatur auf die Wirksamkeit des Wissenschaftsrats beziehen, dann wird dessen autoritative Rolle in der deutschen Wissenschaftspolitik deutlich. Keine andere Einrichtung wird als annähernd einflussreich dargestellt. ${ }^{44}$

Dass das Ausmaß dieser Resonanz vor allem von den Mitgliedern der Wissenschaftlichen Kommission des Wissenschaftsrats nicht immer als zufriedenstellend wahrgenommen wird, wird nicht nur damit zusammenhängen, dass die beschlossenen Empfehlungen in der Praxis, die sie verändern wollen, gar nicht ankommen; auch das ist sicher immer wieder der Fall. Enttäuschungen werden sich bei diesen Wissenschaftlern aber nicht zuletzt daraus ergeben, dass ihre Amtszeiten wesentlich früher zu Ende gehen, als die praktischen Umsetzungen der programmatischen Beschlüsse erfolgen, an denen sie im Wissenschaftsrat beteiligt waren. Auch in der Praxis der Wissenschaften und zumal dann, wenn diese föderalistisch verwaltet und

44 In der großen Mehrheit der 30 auf die deutsche Wissenschaftspolitik bezogenen Beiträge des »Handbuchs Wissenschaftspolitik « (hrsg. von Simon et al. 2010) wird dem Wissenschaftsrat bei zentralen wissenschaftspolitischen Themen ein maßgeblicher Einfluss zugesprochen - beispielsweise bei den Themen Allgemeine Hochschulreform, Fachhochschulen, Hochschulbau, Hochschulräte, Studiengliederung (Bachelor/Master), Nachwuchsförderung (Graduiertenschulen, Assistenzprofessuren), Evaluation (Exzellenzinitiative, Forschungsrating der Fächer, Begutachtung der Ressortforschung, Akkreditierung von Hochschulen und Studiengängen), Nationale Akademie. Im Inhaltsverzeichnis des Handbuchs erhält der Wissenschaftsrat zusammen mit der aus anderen Gründen prominenten Deutschen Forschungsgemeinschaft die bei weitem meisten institutionenbezogenen Nennungen. 
pluralistisch differenziert ist, laufen Evolutionsprozesse träge; sie werden dort, wo sie überhaupt in Gang kommen, durch entgegenstehende Interessen auf ein Tempo heruntergebremst, das kaum noch wahrzunehmen ist - dies umso mehr, je tiefer die Änderungen in die Strukturen des Status quo eingreifen.

Hält man diese Bedingungen im Blick, lässt sich erkennen, dass die strukturell bedeutsamsten Effekte des Wissenschaftsrats sich als Langzeitwirkungen darstellen. Unmittelbare Wirkungen seiner konzeptionellen Empfehlungen zu Entwicklungen des deutschen Wissenschaftssystems sind oft nicht auszumachen; und nicht wenig von dem, was er in über 50 Jahren seiner Tätigkeit empfohlen hat, ist anfangs, manches auch dauerhaft folgenlos geblieben. ${ }^{45}$ In anderen Fällen aber löste der Wissenschaftsrat mit seinen Initiativen Infektionen aus, die erst nach längerer Inkubationszeit zu einem Verfall traditioneller Strukturen und zugleich zu nicht unerheblichen Neustrukturierungen geführt haben. Und dies ist, so belegt die PolitikForschung, nicht ungewöhnlich im politischen Betrieb - schon wegen seiner grundlegenden Abhängigkeit von allgemeinen Ideen und Überzeugungen, deren Wandel sich in der Regel langsam vollzieht. ${ }^{46}$

Entsprechende Karrieremuster lassen sich für die vorgelegten Reformkonzeptionen des Wissenschaftsrats belegen. Ein Beispiel gibt die überaus beschwerliche Entwicklung von Studienreformen, für die der Wissenschaftsrat schon in Empfehlungen des Jahres 1966 eine »Differenzierung des Ausbildungszieles in gestuften Studiengängen " empfahl; den allgemeinen berufsbildenden Abschlüssen sollten für »die befähigten Studenten « Aufbaustudien folgen. ${ }^{47}$ Gegen solche Pläne entwickelte sich eine wirksame Koalition von Opponenten. Ähnliches ergab sich gegen die gleichgerichteten »Empfehlungen zur Struktur des Studiums « Anfang 1986. ${ }^{48}$ Allerdings konnten sich da schon die Vorschläge zur Einführung von Graduiertenkollegs (übrigens "gegen zunächst heftige Grundsatzkritik « der DFG) langsam durchsetzen. Erst Ende der 1990er Jahre begann im Rahmen des europäischen »Bologna-Prozesses « eine Differenzierung des Studiums mit Bachelor- und Masterabschlüssen, die den frühen Wissenschaftsratsvorstellungen im Grundsatz entsprachen. ${ }^{49}$ Die internationale Entwicklung öffnete den national blockierten Programmen die Chance zum späten Erfolg. ${ }^{50}$

Die Differenzierung der Studienabschlüsse erscheint als der lehrbezogene Spezialfall eines allgemeineren Paradigmenwechsels, den der Wissenschaftsrat über Jahr-

$45 \mathrm{Zu}$ letzterem gehören zum Beispiel die bislang vergeblichen Versuche des Wissenschaftsrats, die Fachhochschulen gegenüber den Universitäten aufzuwerten. Es wird interessant sein, zu verfolgen, ob der mit den Empfehlungen zur Rolle der Fachbochschulen im Hochschulsystem im Jahre 2010 erneute Reformversuch am Ende doch Wirkungen zeitigt.

46 Vgl. Héritier 1993, S. 17 ff.; Nullmeier 1993, S. 177 ff.; zur Ökonomie von Langzeiteffekten institutionellen Handelns allgemein Pierson 2004.

47 Siehe Bartz 2007, S. $84 \mathrm{ff}$.

48 Ebd., S. 148 ff.

49 Köhler 2008, S. 12.

50 Vgl. Kingdon 1984, S. 173 ff.

Leviathan, 40. Jg., 2/2012 
zehnte betrieben hat, nämlich die Umwandlung der Humboldt'schen Bildungsuniversität in eine unternehmerische Wettbewerbsuniversität. ${ }^{51}$ Mit diesem Wandel geht die Umstellung eines Gleichheits- auf ein Differenzierungsparadigma der deutschen Hochschulpolitik einher. ${ }^{52}$ Schon in den 1960er Jahren hatte vor allem die Wissenschaftliche Kommission gegen die humboldtianische Einheitsrhetorik und die vorherrschende Kollegialverfassung deutscher Universitäten ein Plädoyer für zunehmend leistungsabhängige Differenzierungen ihrer Strukturen und Akteure entworfen, ein Konzept, das angesichts der gleichzeitigen Forderungen nach mehr Autonomie der Hochschulen mit den in der Verwaltungskommission versammelten Besitzwahrungsansprüchen staatlicher Steuerung zuerst einmal kollidierte und deshalb schon in den internen Beratungen der Vollversammlung stark verwässert wurde. ${ }^{53}$ Auch in der verharmlosten Fassung fanden die 1968er "Empfehlungen zur Struktur und Verwaltungsorganisation der Universitäten « in der Wissenschaftspolitik keine positive Resonanz. Von den Differenzierungsvorschlägen kam allein das schon vorher entworfene Konzept der sogenannten Sonderforschungsbereiche (wieder gegen die Bedenken der DFG) in die Erprobung und wurde bald als »Erfolgsmodell «54 leistungsbestimmter Förderung selektiv, aber in zunehmend größerer Breite und bis heute hin etabliert. Mit den 1975 verabschiedeten »Empfehlungen zu Organisation, Planung und Förderung der Forschung ", vor allem aber mit den 1985 verabschiedeten »Empfehlungen zum Wettbewerb im deutschen Hochschulsystem « nahm der Wissenschaftsrat einen erneuten und dieses Mal sogar radikalisierten Anlauf zur Durchsetzung von Effizienzkriterien im Hochschulsystem. Aber wieder bedurfte es weiterer externer Anstöße, darunter erneut des Drucks internationaler Herausforderungen an die deutsche Wissenschaft, um mit der sogenannten Exzellenzinitiative neue Maßstäbe für eine unternehmerische Universität durchzusetzen. ${ }^{55}$

Richard Münch hat Recht, den inzwischen hegemonial gewordenen Rang des Differenzierungsparadigmas, das zunehmende Ungleichheiten zwischen Universitäten, Fakultäten, Studiengängen und Hochschullehrern erzeugt, vor allem auf das Wirken des Wissenschaftsrats zurückzuführen; er sei - heißt es bei Münch ${ }^{56}$ etwas überzogen hierarchisch - »als Steuerungsinstanz im Machtzentrum des akademischen Feldes « maßgeblich für die Durchsetzung jener »Exzellenzrhetorik « gewesen, die mit der Hochwertung von Begriffen wie Wettbewerb, Profilbildung, Schwerpunktsetzung, Drittmittel, Vernetzung, Cluster, Evaluation und Transparenz das Vokabular für die Legitimierung der tiefgreifenden Reformen des Wissenschafts-

51 Maasen, Weingart 2008; sehr kritisch Münch 2007.

52 Zuletzt WR 2010.

53 Bartz 2007, S. 97 ff.

54 Ebd., S. 99.

55 Neidhardt 2010, S. 58 ff. Eine normative Bedeutung der Referenzgröße »Internationalität « in Wissenschaftsratspapieren belegt Baier (2011, S. 381 f.) am Beispiel des WR-Forschungsratings.

56 Münch 2007, S. 302. 
systems bereitstellt. Der Wissenschaftsrat hat in der Tat die Rahmung der neuen Wissenschaftspolitik langfristig vorbereitet, zunehmend systematisiert, mit Hilfe der DFG wirksam verbreitet ${ }^{57}$ und nach erster Wahrnehmung unerwünschter Nebenwirkungen auch schon nachtariert. ${ }^{58}$ Das heißt nicht, dass er hier und bei anderen Themen die Reformkonzepte, mit denen er wirksam wurde, selbst auch erfunden hätte; es muss nicht sein Ehrgeiz sein, originell zu erscheinen. Seine genuine Leistung kann vielmehr darin bestehen, solche Konzepte aufzunehmen und sie für eine politische Agenda herzurichten. Und ergibt sich daraus ein politischer Einfluss, dann macht dieser sich, das zeigt der Fall der Exzellenzinitiative, nicht unmittelbar exekutiv geltend, er wirkt über allgemeines »Framing « zuerst einmal eher epistemisch und kulturell, und es bedarf einer Fülle von politischen Zusatzbedingungen, um sich am Ende auch durchzusetzen; das kostet Zeit - und das macht letztlich auch die Frage unentscheidbar, welcher Akteur für welche Effekte im strengen Sinne ursächlich war. Jenseits aller Kausalitätsfiktionen lässt sich nur bestimmen, wessen Einfluss, wenn auch nicht unbedingt notwendig und hinreichend, so doch bedeutsam erscheint. Und in dieser Hinsicht ist die Rolle des Wissenschaftsrats für die wichtigsten Entwicklungen der Wissenschaftspolitik generell nicht zu unterschätzen, oft sogar auffällig stark.

Generell eindeutiger erscheint die Rolle des Wissenschaftsrats (2) bei der Durchsetzung der Standards, die er in seinen konzeptionellen Stellungnahmen für die Vermessung wünschenswerter Wissenschaftsentwicklungen postuliert hat. ${ }^{59}$ Durch zahllose Evaluationen von außeruniversitären Forschungseinrichtungen - und auch von Universitäten - und zunehmend bei der Prüfung sogenannter Akkreditierungen von privaten Hochschuleinrichtungen hat der Wissenschaftsrat der Begutachtungspraxis von Wissenschaft hierzulande seine Gütemaßstäbe und » best-practice «Verfahrensregeln entwickelt, festgeschrieben und vermittelt. ${ }^{60}$ Das hat zu flächendeckenden Qualifizierungen sicher erheblich beigetragen, auch wenn Kriterien und Praktiken angesichts unerwünschter Nebeneffekte im Einzelnen umstritten blieben. ${ }^{61}$ Seit 2007 ist es dem Wissenschaftsrat, angeregt und unterstützt vom Haushaltsausschuss des Bundestags, sogar gelungen, in die staatliche Domäne der sogenannten Ressortforschung einzudringen, die sich viele Ministerien zur Unterstüt-

57 WR 2003; WR 2006.

58 WR 2010; WR 2011.

59 Vgl. Bartz 2007, S. 204 ff.

60 Zuletzt WR 2008.

61 Die generelle Akzeptanzbereitschaft ist bei den jeweils von gutachterlichen Urteilen betroffenen Einrichtungen allerdings dadurch gestiegen, dass die »Grundsätze für erfolgreiche Evaluationen (Best Practice) «(WR 2008, S. 289 ff.) den jeweiligen Einrichtungen bestimmte formale Kontrollrechte über den Bewertungsprozess einräumen und ihnen für die Durchführung ihrer Bewertung zudem die Rekrutierung »renommierter Fachgutachter « aus dem In- und Ausland zusichern. Beides ergab sich aus kritischen Einlassungen auf bestimmte Evaluationsfälle. Dass der Wissenschaftsrat darauf so reagierte, wie das der Fall war, lässt sich als ein Beispiel für institutionelles Lernen deuten. 
zung ihrer politischen Arbeit halten. ${ }^{62}$ Hier bleibt vor allem in den jeweils betroffenen Ressorts kontrovers, ob vom Wissenschaftsrat nicht eine Überakademisierung ihrer Einrichtungen befördert wird; ${ }^{63}$ dies umso mehr, als seit einigen Jahren die Verbindlichkeit der Wissenschaftsratseinlassungen bei diesen Einrichtungen ebenso wie bei allen anderen Evaluationen durch die 2003 eingeführte Verpflichtung dahingehend gesteigert wird, »zeitnah, spätestens in drei Jahren, über die Umsetzung der Empfehlungen zu berichten «.

$\mathrm{Zu}$ berücksichtigen ist allerdings auch hier, dass der Wissenschaftsrat selbst keine Sanktionsgewalt besitzt, um die Durchsetzung seiner Vorstellungen machtvoll zu sichern. Entscheidend dafür sind neben den Akteuren wissenschaftlicher Selbstverwaltung, die von ihrer relativen Autonomie profitieren, die politischen Institutionen, die als Geldgeber ihrer Einrichtungen exekutiven Einfluss besitzen. Allerdings erscheinen auch diese nicht unbeeindruckt von dem Prestige des Wissenschaftsrats, mit dem dessen Einlassungen ausgestattet sind; jedenfalls sind im Evaluationsbetrieb des Wissenschaftsrats kaum Fälle bekannt, bei denen kritisierte Forschungseinrichtungen unbekümmert ihre alte Praxis weitertreiben.

Hier kommt ein Mechanismus ins Spiel, der den institutionellen Kern des generellen Einflusses des Wissenschaftsrats ausmacht: Die Reputation des Wissenschaftsrats ist so groß, dass er autorisiert erscheint, über die Reputation anderer Wissenschaftseinrichtungen zu entscheiden - und dies nach Gütemaßstäben, die er selbst definiert hat. Solange das Label "Wissenschaft " auch in der Öffentlichkeit einen attraktiven Marktwert besitzt ${ }^{64}$ und solange die Mitgliedschaft in der Scientific Community als ehren- und deshalb auch als erstrebenswert gilt, solange funktioniert das, was Gunnar Folke Schuppert am Beispiel anderer »Reputationsgemeinschaften « als "governance by reputation « bezeichnet hat. ${ }^{65}$ Die Gesellschaft

62 Gegen Interventionen des Wissenschaftsrats erfolgreich immunisiert sind nicht nur die umfangreiche private Industrieforschung und die sogenannten Großforschungseinrichtungen des Bundes, sondern mit Bezug auf eigene Evaluationspraktiken und mit dem robusten Selbstbewusstsein einer international erfolgreichen Forschungsorganisation auch die Max-Planck-Gesellschaft.

63 Gegen diese Annahme könnte sprechen, dass der Wissenschaftsrat im Hinblick auf außerakademische Außeneinflüsse auf Ressortforschungseinrichtungen, die vor allem aus politisch betroffenen Wirtschaftsbereichen versucht werden, bislang relativ zurückhaltend blieb. Obwohl zum Beispiel im Falle des begutachteten Umweltbundesamtes (WR 2007, S. 272-348) 37 Prozent der Drittmittel aus der Wirtschaft kamen und 58 Prozent der vergebenen Aufträge an die Wirtschaft gingen (ebd., S. 344 f.), findet sich im Bewertungsbericht keinerlei Stellungnahme über die Besonderheiten der damit eingegangenen Bindungen. Auch wenn diese für die Autonomie der Wissenschaft in Ressortforschungseinrichtungen als unproblematisch eingeschätzt werden könnten, hätte man das doch gern gewusst. - Über diesen Fall hinaus entsteht der Eindruck, dass der Wissenschaftsrat das Autonomieproblem der Wissenschaft durchweg noch im Verhältnis Politik/Wissenschaft sucht und die mit dem Grassieren von "Public-private Partnership " aufkommenden Probleme einer ökonomischen Unterwanderung jener Disziplinen vernachlässigt, die marktnah und gewinnträchtig operieren.

64 Neidhardt 2004, S. 321-330.

65 Schuppert 2010, S. 94. 
reguliert sich nicht nur über Macht- und Besitz-, sondern auch über Prestigedifferenzierungen. Auch bei einem Mangel an Zwangsmitteln wirkt allein die symbolische Auf- oder Abwertung - zum Beispiel die Veröffentlichung von Ratings und Rankings, wenn sie von einer Institution vollzogen wird, die im Hinblick auf Kompetenz und Integrität Vertrauen genießt. Umgekehrt lässt sich folgern: Dass dies in der Wissenschaft im Falle des Wissenschaftsrats offenkundig wirkt, spricht für seine eigene Reputation. Er bewährt sich in dem Maße, in dem er den Status einer Reputationsinstanz gewinnt.

\section{Verallgemeinerungen}

Anlass zu der hier vorgelegten Analyse gab die Frage, wodurch nicht nur das lange Überdauern des Wissenschaftsrats, sondern auch seine tatsächlichen Wirkungen im Felde der Wissenschaftspolitik erklärbar seien, wenn doch deutlich ist, dass das notorische Instrumentarium politischen Erfolgs, nämlich der Besitz eigener Zwangsmittel, hier nicht unmittelbar verfügbar ist. Nützlich für die Analyse erwies sich der Anschluss an die Gehlen'sche Formel von der »Überdeterminiertheit einer dauerhaften Institution ", welche die instrumentellen und kulturellen Bedingungen für das Entstehen von Reputation als sozial wirksamer Ressource besonderer Art in den Blick nimmt. Fasst man am Schluss die vorgelegten Beobachtungen und Deutungen des Wissenschaftsrats mit dem Ziel zusammen, sie zu verallgemeinern, dann eröffnen sich fruchtbare Perspektiven mit dem Bezug auf jene institutionellen Kontexte, die in der Tat die angesprochene Steuerung durch Reputation, eine "governance by reputation «, zustande bringen.

Ein passender Verallgemeinerungsansatz lässt sich mit der Zuordnung des Wissenschaftsrats zu einem Institutionentyp finden, der zuerst im Bereich der internationalen Politik mit dem Begriff »epistemic authority « belegt wurde. ${ }^{66}$ Dort wurden neue transnational wirksame " epistemic communities « als Netzwerke von "professionals with recognized expertise and competence in a particular domain and an authoritative claim to policy relevant knowledge within that domain or issue area " bezeichnet. ${ }^{67}$ Beispiele lieferten Greenpeace und Amnesty International, aber auch das »Intergovernmental Panel on Climate Change « (IPCC) und der »International Board for Accounting Standards «. »Epistemische Autorität beruht auf Fachwissen und moralischer Autorität. In diesem Fall werden die Ansichten und die Position eines Individuums oder einer Institution übernommen, weil sie als kundig und gleichzeitig unparteiisch erscheinen ... Es ist [dabei] nicht die Qualität der spezifischen Argumentation, sondern die allgemeine Reputation einer Institution oder Person, die entscheidend ist. «68 Es handelt sich um die Effekte generalisierter Ver-

66 Ich verdanke einen entsprechenden Hinweis Michael Zürn (vgl. auch Zürn et al. 2011; Zürn 2012).

67 Haas 1990, S. 3.

68 Zürn 2012, S. 13.

Leviathan, 40. Jg., 2/2012 
trauenswürdigkeit, die auch Fehlertoleranzen begründet: Vertrauenswürdigkeit muss sich nicht in jedem Einzelfall beweisen. ${ }^{69}$

Dieser Ansatz lässt sich, wie Zürn ${ }^{70}$ zeigt, auch auf nationale Einrichtungen beziehen und dabei mit Bezug auf allgemeine Legitimationsprobleme politischen Handelns, die mit ihnen verbunden sind, systematisieren - zum Beispiel bei Einrichtungen wie Gerichten, speziell des Verfassungsgerichts; Ethikkommissionen wären wohl ebenfalls einschlägig, sicher auch die Kirchen, in gewisser Weise vielleicht das Amt des Bundespräsidenten. Gemeinsam ist ihnen die Chance, programmatische Orientierungen, institutionelle Standards und kognitive Muster durchzusetzen, insoweit ihnen Kompetenz und Vertrauenswürdigkeit zugeschrieben wird. Sie lassen sich mit ihrer epistemischen Autorität als Reputationsinstanzen begreifen. Sie wirken in Analogie zu den Geldmechanismen sozialen Einflusses gewissermaßen als Reputationsbanken, die in einem gesellschaftsweiten Prestigehandel imstande sind, anderen Institutionen und Personen (auch Dingen und Prozessen) Reputation zu verleihen oder zu entziehen - und das wirkt, weil der Besitz von Reputation ein generalisiertes Medium darstellt, das sich in gewissen Grenzen in andere Einflusssorten konvertieren lässt - beispielsweise in Geld und Macht; ${ }^{71}$ es lohnt sich also, als reputierlich ausgewiesen zu werden.

Interpretiert man im analytischen Bezugsrahmen einer Theorie epistemischer Autorität den besonderen Fall des Wissenschaftsrats für das Gelingen von Reputationseinflüssen, erweisen sich auch über seinen speziellen Fall hinaus mehrere Umstände als folgenreich. Solche Autoritäten funktionieren (1) nur in Abhängigkeit von bestimmten externen Bedingungen des politischen Systems, in dem sie sich entwikkeln. Sie entstehen und bewähren sich - wie der Wissenschaftsrat - in politischen Verhältnissen, deren Pluralismus sich hierarchisch nicht hinreichend regulieren lässt. Sie erzeugen für die Lösung systemrelevanter Probleme einen institutionellen Verständigungsbedarf. Dieser ergibt sich in den postnationalen Konstellationen des internationalen Feldes für globale Probleme, die sich nicht durch Machtworte einer imperialen Zentralinstanz entscheiden lassen. Sie entstehen in den nationalen Politikfeldern der Bundesrepublik zum Beispiel für die Belange der Wissenschaft sowohl durch den Föderalismus der Wissenschaftspolitik als auch durch die stark ausgeprägten Autonomien der Wissenschaft selber. Bund, Länder und Wissenschaft suchen eine Arena, in der sich die betroffenen Parteien begegnen und abstimmen kön-

69 Hans Vorländer über die Wirkungsweise des Bundesverfassungsgerichts: „Ein Verfassungsgericht verfügt nicht per se über die Sanktionsmittel wie beispielsweise Gesetzgebung und vollziehende Gewalt, um seine Entscheidungen durchzusetzen ... Über eine Folge von zustimmungsfähigen Entscheidungen baut sich ein generalisiertes Vertrauen in die Institutionen der Verfassungsgerichtsbarkeit auf, das nicht mehr allein von der konkreten Spruchpraxis abhängt «. Es erscheint generalisiert dadurch, dass es »kurzfristige Erschütterungen und Akzeptanzverweigerungen zu absorbieren vermag « (Vorländer 2011, S. 18, S. 21). Auch der Wissenschaftsrat bedarf nicht des Nimbus der Unfehlbarkeit, um vertrauenswürdig zu erscheinen.

70 Zürn 2012.

71 Vgl. Parsons 1980. 
nen. Das "höhere Interesse" (Gehlen) des Gemeinwesens wird bedient, wenn eine solche Arena in einer Einrichtung wie dem Wissenschaftsrat tatsächlich entsteht; dass in ihr Verständigungen zum Wohl der Wissenschaft gelingen, ist seine »Leitidee« (Hauriou).

Es kann dann auch nicht überraschen, wenn der institutionelle Vermittlungsbedarf (2) eine entsprechende politische Nachfrage nach einer epistemischen Autorität auslöst, welche mit konzeptionellen Entwürfen und gutachtlichen Kontrollen Vermittlungen im Politikbereich betreibt. Dies ist im Falle des Wissenschaftsrats mit einem zwar kündbaren, aber politisch stabilen »Verwaltungsabkommen " geschehen. Der Wissenschaftsrat besitzt keine eigenen exekutiven Durchgriffsrechte, aber er gehört zu den besonderen Fällen, in denen »eine epistemische Autorität als solche durch politische Institutionen gleichsam ernannt «72 und unterstützt und dann auch deren robusteren Herrschaftskompetenzen angeschlossen wird. ${ }^{73}$ Die Wirkung seiner Reputation wird in diesem Fall dadurch gesteigert, dass als "Deckungsreserve " die Macht- und Geldmittel der politischen Instanzen einspringen können, zumal diese im Wissenschaftsrat selber einflussreich vertreten sind. Anders als reine Politikberatungsgremien ermöglicht der Wissenschaftsrat Vorabstimmungsfunktionen zwischen Bund und Ländern sowie zwischen Politik und Wissenschaft. Der politische Auftrag verleiht dem Auftragnehmer eine gesteigerte formelle Legitimation.

In welchem Maße eine intermediäre Institution sich mit Verständigungsleistungen tatsächlich bewährt, hängt (3) auch davon ab, ob und in welcher Weise ihre internen Prozesse unter einen institutionalisierten Verständigungsdruck gestellt werden und dadurch zu integrativen Leistungen angehalten wird. Im Falle des Wissenschaftsrats als einer hybriden Einrichtung von Politik und Wissenschaft ist dieser Druck (anders als bei dem homogener verfassten Bundesverfassungsgericht) zu einem Konsenszwang radikalisiert: Keine der Parteien kann die andere überstimmen, weder die Politik die Wissenschaft noch die Wissenschaft die Politik. Zu den Folgekosten dieser starken Regelung gehört die Selektivität der Themen, die auf die Agenda der Einrichtung geraten. Regelmäßig läuft die Suche nach Themen, die zwischen den Parteien konsentierbar erscheinen, verbunden mit dem Ausschluss solcher Themen, für die das nicht erwartet werden kann - auch wenn diese als wichtig erscheinen. Hier macht sich eine weitere Abhängigkeit von den politischen Außenbedingungen bemerkbar, die eine "governance by reputation " prägen. Ist die Zahl der Themen in einem politischen System, zu denen die beteiligten Parteien ihre Positionen polarisiert und einander insoweit unverträglich gemacht haben, sehr groß, schwinden die Chancen epistemischer Autoritäten, über Reputation Einfluss auszuüben. Ihre Reputation bricht sich an den Interessengegensätzen des politischen Feldes, die über das Format »dosierter Diskrepanz (Heckhausen) hinausgehen.

72 Zürn 2012, S. 14.

73 Der Reputationseffekt läuft in diesem Fall mehrstufig, nämlich zuerst innerhalb des Wissenschaftsrats mit dem Einfluss der Wissenschaftlichen Kommission auf die Verwaltungskommission, sodann mit deren Unterstützung als Wissenschaftsratseffekt auf die operative Wissenschaftspolitik. 
Ein Reputationsregime bewährt sich bei politischen Themen im mittleren Dissensbereich politischer Interessen. Im Falle dosierter Diskrepanzen können Verständigungsprozesse funktionieren, und finden sie zudem unter Bedingungen eines institutionalisierten Konsenszwangs statt, kann epistemische Autorität (4) von einem »deliberative setting " (Jon Elster) qualifiziert werden, da sich dann alle darauf einlassen müssen, zu argumentieren anstatt bloß zu verlautbaren. Die »Herstellung eines gemeinsamen Interpretationsrahmens « (Ulla Foemer) für anstehende Probleme setzt voraus, dass man sich wechselseitig überzeugt. Finden mit diesem Ziel Deliberationen statt und führen diese zu gemeinsamen Entscheidungen, dann entsteht in den Augen des Publikums die schöne Aura des »Runden Tisches", an den sich die Beteiligten setzen und so miteinander reden, dass etwas dabei herauskommt. Letzteres ist im vorliegenden Fall dem Wissenschaftsrat in einer erheblichen Zahl von Fällen gelungen - und zwar, im politischen Feld nicht durchweg üblich, mit sehr geringer Lärmentwicklung; auch hier ist die diskret funktionierende Geschäftsstelle des Wissenschaftsrats von erheblicher Bedeutung. ${ }^{74}$

Dass das, was herauskommt, in der Praxis, auf die es sich bezieht, auch ankommt und, wenn nicht kurzfristig, so doch langfristig in einer hinreichenden Zahl von Fällen jene Wirkungen erzeugt, die beabsichtigt und gewünscht sind, setzt nicht nur das Gelingen von Konsens innerhalb der Institution und die damit entstehende politische Rückendeckung voraus. Es bedarf (5) zusätzlicher Mechanismen von Responsivität, um zumindest im wissenschaftspolitisch relevanten Publikum Akzeptanz zu erzeugen. Dafür stehen epistemischen Autoritäten je nach den sozialen und politischen Kontexten, in denen sie wirken, unterschiedliche Verfahren zur Verfügung. Am Wissenschaftsrat lässt sich erkennen, dass akzeptanzsichernde Legitimitätsbedingungen auf verschiedenen Ebenen mit unterschiedlichen Legitimationsleistungen hergestellt werden können. Die in der Verwaltungskommission repräsentierte Politik von Bund und Ländern sorgt, im Wissenschaftsrat nicht überstimmbar, (a) für ein gewisses, allerdings limitiertes Maß an demokratischer Grundlegitimität der Einrichtung selbst. Die Art der Rekrutierung reputierlicher Wissenschaftler aus dem Gesamtspektrum der Disziplinen in die Wissenschaftliche Kommission, ebenfalls im Wissenschaftsrat nicht überstimmbar, verschafft dem Wissenschaftsrat (b) ein belastbares Maß an Vertrauenswürdigkeit, stellt Reputation in der Wissenschaft doch das resonanzbestimmende Kapital von Personen und Einrichtungen dar. ${ }^{75}$ Die Wissenschaftsgemeinschaft ist eine Reputationsgemeinschaft im Schuppert'schen Sinne, und man kann annehmen, dass insoweit nicht nur die Wissenschaft, sondern auch die Wissenschaftspolitik einer Steuerung durch Reputation in einem besonderen Maße zugänglich ist. Aber ausdifferenzierte Institutio-

74 Im Falle des Wissenschaftsrats ist leicht ist zu übersehen, dass seine Geschäftsstelle schon aus Eigeninteresse am Funktionieren und am Fortbestand ihres Arbeitsgebers immer wieder die »broker«-Rolle eines stillen Maklers zwischen den Fronten spielt. Anzunehmen ist, dass »Sekretariate « dieser Art auch für andere Institutionen wichtiger sind, als sie sich selber, wenn sie gut sind, den Anschein geben; sie erscheinen als die »Knoten « im Netzwerk ihrer Organisation, ebenso unscheinbar wie bedeutsam.

75 Luhmann 1970, S. $236 \mathrm{ff}$. 
nen, die überdies nur begrenzt öffentlich transparent sind, stellen in der Regel Eliteprojekte dar, die gut daran tun, mit (c) zusätzlicher Responsivität für Basisbindungen an ihre Klientel zu sorgen. In der Hinsicht hat der Wissenschaftsrat in seinen Verfahren zunehmend Aufmerksamkeit »nach unten « routinisiert. Dazu gehört die Inklusion der sehr zahlreichen wissenschaftlichen Experten, die für die Bearbeitung der wechselnden Aufgaben des Wissenschaftsrats für Sachverstand sorgen. Ein partizipatives Element ist im Falle gutachtlicher Festlegungen überdies dadurch routinisiert, dass die jeweils Betroffenen sowohl gegen die Wahl der Gutachter als auch gegen deren Sachstandsbericht Einspruch einlegen können. Auf diese Weise sind Möglichkeiten dafür nutzbar, dass die Exklusivität einer epistemische Autorität nicht zu ihrer politischen und sozialen Isolation führt. Dass der Wissenschaftsrat sich solche Möglichkeiten entwickelte, ist Teil eines institutionellen Lernens, das zu seiner »Ultrastabilität « beigetragen hat. ${ }^{76}$

Auch wenn diese institutionellen Konstellationen einer epistemischen Autorität Legitimationen und Wirkungschancen vermitteln, bedarf es am Ende (6) doch noch des Glücksfalls von Personen, die imstande sind, mit Augenmaß, taktischem Geschick und einem Sinn für angemessenes Timing Spielräume zu nutzen und Grenzen zu respektieren. Es sollte ihnen auch nicht an Frustrationstoleranz mangeln, um Enttäuschungen und Kritik produktiv wenden zu können. Ein »akteursszentrierter Institutionalismus " 77 verweist, indem er den Blick auf die Akteure freigibt, auch auf deren Idiosynkrasien, die sich schwerlich wegrationalisieren, wohl aber nutzen lassen. Deren Rolle lässt sich im vorliegenden Falle auch an der starken Bedeutung ermessen, den in der Geschichte des Wissenschaftsrats einzelne Vorsitzende sowie einige Generalsekretäre gewonnen haben. Das wird in anderen Institutionen, so sie denn erfolgreich erscheinen, ähnlich sein.

\section{Literatur}

Baier, Christian 2011. »Wissenschaft regieren - Eine diskursanalytische Studie zum Forschungsrating des Wissenschaftsrates", in Soziale Welt 62, S. 371-390.

Bartz, Olaf 2007. Der Wissenschaftsrat. Entwicklungslinien der Wissenschaftspolitik in der Bundesrepublik Deutschland 1957-2007. Stuttgart: Steiner.

Benz, Winfried 1996. „Der Wissenschaftsrat ", in Handbuch des Wissenschaftsrechts, Bd. 2, hrsg. v. Flämig, Christian, S. 1667-1687. 2. Aufl. Berlin: Springer.

Braun, Dietmar 1997. Die politische Steuerung der Wissenschaft. Frankfurt a. M., New York: Campus.

Buckley, Walter 1967. Sociology and modern systems theory. Englewood Cliffs: Prentice Hall.

Burchardt, Ulla 2011. »Kinder sind eine nationale Aufgabe «, in Der Tagesspiegel, 16. November 2011, S. 6.

Cadwallader, Mervyn L. 1969. »Die kybernetische Analyse des Wandels", in Theorien des sozialen Wandels, hrsg. v. Zapf, Wolfgang, S. 141-146. Berlin: Kiepenheuer \& Witsch.

Degenkolbe, Gert 1965. »Über logische Struktur und gesellschaftliche Funktionen von Leerformeln «, in Kölner Zeitschrift für Soziologie und Sozialpsychologie 17, S. 327-337.

76 Cadwallader 1969; vgl. Buckley 1967, S. 58 ff.

77 Mayntz, Scharpf 1995.

Leviathan, 40. Jg., 2/2012 
Elster, Jon 1986. »The market and the forum: three varieties of political theory «, in Foundations of political theory, hrsg. v. Elster, Jon; Hylland, Aamund, S. 103-132. Cambridge: Cambridge University Press.

Elster, Jon 1989 a. »Introduction «, in Deliberative democracy, hrsg. v. Elster, Jon, S. 1-18. Cambridge: Cambridge University Press.

Elster, Jon 1989 b. »Deliberation and constitution making ", in Deliberative democracy, hrsg. v. Elster, Jon, S. 97-122. Cambridge: Cambridge University Press.

Foemer, Ulla 1981. Zum Problem der Integration komplexer Sozialsysteme am Beispiel des Wissenschaftsrats. Berlin: Duncker \& Humblot.

Gehlen, Arnold 1957. Die Seele im technischen Zeitalter. Hamburg: Rowohlt.

Haas, Peter 1990. "Epistemic communities and international coordination", in International Organization 46, 1, S. 1-35.

Habermas, Jürgen 1992. "Deliberative Politik - ein Verfahrensbegriff der Demokratie «, in Faktizität und Geltung. Beiträge zur Diskurstheorie des Rechts und des demokratischen Rechtsstaates, Habermas, Jürgen, S. 349-398. Frankfurt a. M.: Suhrkamp.

Hauriou, Maurice 1965. Die Theorie der Institution und zwei andere Aufsätze. Berlin: Duncker \& Humblot.

Héritier, Adrienne 1993. »Einleitung: Policy-Analyse. Elemente der Kritik und Perspektiven der Neuorientierung ", in Policy-Analyse. Kritik und Neuorientierung, hrsg. v. Héritier, Adrienne, S. 9-36. Opladen: Westdeutscher Verlag.

Kaase, Max 1999. »Politik im Zwiespalt zwischen Wandel und Status Quo. Eine Analyse im Hinblick auf den deutschen Wissenschaftsrat ", in Eigenwilligkeit und Rationalität sozialer Prozesse, hrsg. v. Gerhards, Jürgen; Hitzler, Roland, S. 234-257. Opladen, Wiesbaden: Westdeutscher Verlag.

Kielmansegg, Peter Graf 1990. »Notizen zum Thema Wissenschaftsrat", in Verwalten ist Gestalten. Festschrift für Arnim Danco, hrsg. v. Kleiner, Ulrich, S. 211-219. Düsseldorf: Triltsch.

Kielmansegg, Peter Graf 1998. "Ansprache zum Festakt Vierzig Jahre Wissenschaftsrat ", in Wissenschaftsrat 1957-1997, hrsg. v. Wissenschaftsrat. Köln: Geschäftsstelle des Wissenschaftsrats.

Kingdon, John W. 1984. Agendas, alternatives, and public policies. Michigan: Harper Collins.

Köhler, Horst 2008. Festansprache zur 50-Jahr-Feier des Wissenschaftsrats 2007, hrsg. v. Wissenschaftsrat. Köln: Geschäftsstelle des Wissenschaftsrats.

Leibfried, Stephan. Hrsg. 2010. Die Exzellenzinitiative. Zwischenbilanz und Perspektiven. Frankfurt a. M.: Campus.

Lepsius, M. Rainer 1990. »Die Prägung der politischen Kultur der Bundesrepublik durch institutionelle Ordnungen ", in Interessen, Ideen und Institutionen, Lepsius, M. Rainer, S. 63-84. Opladen: Westdeutscher Verlag.

Luhmann, Niklas 1970. "Selbststeuerung der Wissenschaft", in Soziologische Aufklärung. Aufsätze zur Theorie sozialer Systeme, Luhmann, Niklas, S. 232-252. Köln, Opladen: Westdeutscher Verlag.

Maasen, Sabine; Weingart, Peter 2008. »Unternehmerische Universität und neue Wissenschaftskultur ", in Wissenschaft unter Beobachtung, hrsg. v. Matthies, Hildegard; Simon, Dagmar, S. 141-160. Wiesbaden: VS Verlag für Sozialwissenschaften.

Mayntz, Renate; Scharpf, Fritz W. 1995. »Der Ansatz des akteurzentrierten Institutionalismus «, in Gesellschaftliche Selbstregelung und politische Steuerung, Mayntz, Renate; Scharpf, Fritz W., S. 39-72. Frankfurt a. M.: Campus.

Münch, Richard 2007. Die akademische Elite. Zur sozialen Konstruktion wissenschaftlicher Exzellenz. Frankfurt a. M.: Suhrkamp.

Neidhardt, Friedhelm 2004. »Wissenschaft als Politikum - Öffentlichkeitsbedürfnisse der Forschung auf dem Prüfstand ", in Die Stimme der Medien. Pressekommentare und politische Öffentlichkeit in der Bundesrepublik, Eilders, Christiane; Neidhardt, Friedhelm; Pfetsch, Barbara, S. 312-335. Wiesbaden: VS Verlag für Sozialwissenschaften.

Neidhardt, Friedhelm 2010. »Exzellenzinitiative - Einschätzungen und Nachfragen ", in Die Exzellenzinitiative. Zwischenbilanz und Perspektiven, hrsg. v. Leibfried, Stephan, S. 53-84. Frankfurt a. M.: Campus.

Nullmeier, Frank 1993. »Wissen und Policy-Forschung ", in Policy-Analyse. Kritik und Neuorientierung, hrsg. v. Héritier, Adrienne, S. 175-196. Wiesbaden: Westdeutscher Verlag. 
Parsons, Talcott 1980. Zur Theorie der sozialen Interaktionsmedien. Opladen: Westdeutscher Verlag.

Pierson, Paul 2004. Politics in time. History, institutions, and social analysis. Princeton: Princeton University Press.

Röhl, Hans Christian 1994. Der Wissenschaftsrat. Kooperation zwischen Wissenschaft, Bund und Ländern und ihre rechtliche Determinanten. Baden-Baden: Nomos.

Schuppert, Gunnar Folke 2010. Staat als Prozess. Eine staatstheoretische Skizze in sieben Aufzügen. Frankfurt a. M.: Campus.

Simmel, Georg 1968. Soziologie. Untersuchungen über die Formen der Vergesellschaftung. 5. Aufl. Berlin: Duncker \& Humblot.

Simon, Dagmar; Knie, Andreas; Hornbostel, Stefan. Hrsg. 2010. Handbuch Wissenschaftspolitik. Wiesbaden: VS Verlag für Sozialwissenschaften.

Simon, Dieter 1991. » Wie im Westen so auf Erden. Die alte Wissenschaftsroutine lähmt die neuen Länder ", in: Frankfurter Allgemeine Zeitung, 19. März 1991.

Strohschneider, Peter 2007. »Eröffnung des Festakts 50 Jahre Wissenschaftsrat am 5. September 2007 ", in Dokumentation, hrsg. v. Wissenschaftsrat, S. 5-9. Köln: Geschäftsstelle des Wissenschaftsrats.

Stucke, Andreas 2006. "Der Wissenschaftsrat", in Handbuch Politikberatung, hrsg. v. Falk, Svenja et al., S. 248-254. Wiesbaden: VS Verlag für Sozialwissenschaften.

Stucke, Andreas 2010. "Staatliche Akteure in der Wissenschaftspolitik ", in Handbuch Wissenschaftspolitik, hrsg. v. Simon, Dagmar; Knie, Andreas; Hornbostel, Stefan, S. 363-377. Wiesbaden: VS Verlag für Sozialwissenschaften.

Stucke, Andreas 2011. »Quality assurance through procedures - policy advice by the German Science Council «, in The politics of scientific advice, hrsg. v. Lentsch, Justus; Weingart, Peter, S. 199-213. Cambridge: Cambridge University Press.

Thies, Erich 2011. "Viele Eltern haben Panik « (Interview), in DER SPIEGEL, 29. September 2011.

van den Daele, Wolfang 1996. »Objektives Wissen als politische Ressource: Experten und Gegenexperten im Diskurs", in Kommunikation und Entscheidung. Politische Funktionen öffentlicher Meinungsbildung und diskursiver Verfahren. WZB-Jahrbuch 1996, hrsg. v. van den Daele, Wolfgang; Neidhardt, Friedhelm, S. 297-326. Berlin: edition sigma.

Verwaltungsabkommen 2008. Verwaltungsabkommen zwischen Bund und Ländern über die Errichtung eines Wissenschaftsrates vom 5. September 1957 in der Fassung ab 1. Januar 2008.

Vorländer, Hans 2011. »Regiert Karlsruhe mit? Das Bundesverfassungsgericht zwischen Recht und Politik ", in Aus Politik und Zeitgeschichte 61, 35/36, S. 15-23.

Weingart, Peter; Lentsch, Justus 2008. Wissen, Beraten, Entscheiden. Form und Funktion wissenschaftlicher Politikberatung in Deutschland. Weilerswist: Velbrück.

WR (Wissenschaftsrat) 2003. Strategische Forschungsförderung. Empfehlungen zur Kommunikation, Kooperation und Wettbewerb im Wissenschaftssystem. Drs. 5654/03. Essen: Wissenschaftsrat.

WR 2006. Empfehlungen zur künftigen Rolle der Universitäten im Wissenschaftssystem. Drs. 7067-06. Berlin: Wissenschaftsrat.

WR 2007. Empfehlungen und Stellungnahmen 2007, Bd. II. Köln: Geschäftsstelle des Wissenschaftsrats.

WR 2008. Empfehlungen und Stellungnahmen 2008, Bd. III. Köln: Geschäftsstelle des Wissenschaftsrats.

WR 2008 a: »Sachverständige, die im Jahr 2008 an Ausschüssen und Arbeitsgruppen mitgewirkt haben ", in: Empfehlungen und Stellungnahmen 2008, Bd. III, S. 677-685.

WR 2010. Empfehlungen zur Differenzierung der Hochschulen. Drs. 10387-10. Lübeck: Wissenschaftsrat.

WR 2011. Empfehlungen zur Bewertung und Steuerung von Forschungsleistung. Drs. 1656-11. Halle an der Saale: Wissenschaftsrat.

Zürn, Michael 2012. Legitimität und Autorität in der postnationalen Konstellation. Unveröffentlichtes Manuskript. Berlin.

Zürn, Michael; Binder, Martin; Ecker-Ehrhardt, Matthias 2011. International political authority and its politization. Paper, präsentiert auf der 3. Offenen Sektionstagung Internationale Politik der DVPW. Berlin.

Leviathan, 40. Jg., 2/2012 
Zusammenfassung: Der Deutsche Wissenschaftsrat ist eine einflussreiche Politikberatungskommission im föderalistischen System deutscher Wissenschaftspolitik. Sein Einfluss ergibt sich vor allem aus der Reputation, die er sich erworben hat (»governance by reputation «). Verantwortlich dafür sind unter anderem die Art der Rekrutierung und Zusammensetzung seiner Mitglieder aus Politik und Wissenschaft, die Bindung der Entscheidungen an deren Konsens sowie die strategisch selektive Auswahl der Themen, über die er befindet.

Stichworte: Wissenschaftspolitik, Institution, Deliberation, Reputation

\section{Institution, organisation, interaction - functional prerequisites of the German Science Council (Wissenschaftsrat)}

Summary: The German Science Coucil is an established institution in the federal system that manages science policy. Its influence is due primarily to its high reputation ( «governance by reputation «). This is attributable in part to the recruitment and composition of its membership from the fields of politics and science, the consensus rule for decisionmaking, and the strategically restricted selection of issues that it deals with.

Keywords: Science policy, institution, deliberation, reputation

Autor

Prof.em. Dr. D.h.c. Friedhelm Neidhardt

Wissenschaftszentrum Berlin für Sozialforschung

Reichpietschufer 50

10785 Berlin

Email: FrNeidhardt@aol.com 